\title{
An intelligent model for predicting the effect of some effective parameters on anti-surge control in centrifugal compressors
}

\begin{abstract}
To prevent the destructive surge phenomenon in centrifugal compressors, we usually choose the surge control line in a prudent manner, which in turn cause in reducing the efficiency of compressor and results in increasing energy consumption of its actuator. It requires a roughly accurate model of system performance to design an optimum controller. In this paper we introduce a model based on artificial neural networks. It gets the compressor RPM, anti-surge valve state, temperatures, compressor gas output flow rate and its upstream pressure as inputs. We have used the measurements conducted by cybernetics laboratory of Norway University from a centrifugal compressor installed in a gas processing plant as our dataset. We have achieved better results in predicting the system output in comparison to mathematical model based on state equations.
\end{abstract}

Keywords: centrifugal compressor, surge control, anti-surge valve, artificial neural network
Volume 3 Issue 3 - 2017

\author{
Amin Zadeh Shirazi, ${ }^{1,3}$ Majid Hatami, ${ }^{2}$ Ahmad \\ PishroAs| $\left.\right|^{3}$ \\ 'Department of Artificial Intelligence, Islamic Azad University, \\ Iran \\ ${ }^{2}$ Department of Artificial Intelligence, Tabriz University, Iran \\ ${ }^{3}$ Department of Information and Communication Technology, \\ National Iranian Gas Company, Iran
}

Correspondence: Amin Zadeh Shirazi, Department of Artificial Intelligence, Islamic Azad University, Iran,

Email amin.zadeshirazy@gmail.com

Received:September 15, 2017 | Published: October 30, 2017

\section{Introduction}

Centrifugal compressors are widely used in oil and gas industries In order to achieve maximum profit, compressors should be used at maximum efficiency and with the least possible power consumption. The working range of centrifugal compressors is limited due to their flow rate. High flow rate cause in reducing compressor pressure ratio which can leads to choking. On the other hand, if the flow rate is less than a certain threshold, it will cause surge phenomenon in the compressor. In surge state, pressure and flow rate of output gas fluctuate, which can cause vibration in accessories such as pipes, fittings, etc., and can lead to serious damage to facilities and equipment. ${ }^{1}$ There are two ways to deal with the surge phenomenon: controlling active surge and controlling anti-surge. The first method is trying to stabilize the surge, while the second method prevents the compressor working point from reaching the surge line and keeps it permanently to the right side of the line. ${ }^{2}$ In Figure 1, characteristic curves of a typical centrifugal compressor can be seen. In this diagram, the vertical axis shows the compressor output pressure ratio to its input pressure and the horizontal axis shows the flow rate and parallel curves of different compressor RPMs. There is a "surge line" for each compressor that shows the values of the flow rates and the RPMs that cause the compressor to surge. "Surge control line" specifies the boundary of the area that anti-surge controller allows the compressor to operate. The area to the right of this line is the stable operation area of the compressor.

As seen in the Figure 1, a safety margin between the surge line and the surge control line is considered. To prevent surge phenomenon, a return path for the compressor is considered. The compressed gas in the compressor output can return from this path to its input. Anti-surge controller controls the return gas flow using anti-surge valve installed on this path. With this method, in the event of a shortage of input flow to the system, the necessary flow to prevent compressor surge can be provided by opening anti-surge valve. Although this method is not optimal in terms of power consumption, it is widely used in industrial compressors due to its reliability. Due to the damaging effects that a compressor surge can cause, anti-surge controllers choose a large safety margin between surge line and surge control line in a prudent manner. Obviously, by reducing this safety margin, the compressor in a steady RPM can be used to produce higher pressure ratio with more efficiency, thereby power consumption is reduced and profitability is increased. Designing and evaluating better controllers requires a fairly accurate model of system performance. In Jiang ${ }^{3}$ modeling of centrifugal compressors to have active control on surge has been discussed. However, less research has been done on compressor modeling, in a gas pressure boost system to control anti-surge, along with its lateral elements. In Cortinovis ${ }^{4}$ this subject is considered by presenting a model based on state equations. In this paper, we have presented a model based on artificial neural networks and compared our results with the reported results in. ${ }^{5}$ Artificial neural networks have been widely used in various areas of modeling. For example ${ }^{6}$ has discussed different aspects of modeling and analysis of gas turbines operation, and ${ }^{7}$ has used neural networks for modeling, controlling and optimization in corrosion phenomenon. In the following, we introduce artificial neural networks briefly and then examine the studied gas pressure boost system. Finally, we introduce the proposed intelligent model and bring simulation results. The final part of this article is dedicated to the conclusions and future works.

\section{Material \& methods}

\section{Artificial neural networks}

The ultimate goal of introducing artificial neural networks is to simulate the human brain to solve real complex problems. A multilayer perception neural network model is an interconnected array of artificial neurons that are arranged in several layers. Synthetic neurons are a simple model of real nerve cells. Mathematically, the neuron is a function with multiple inputs and an output. As shown in Figure 2, the inputs are summed up after being multiplied by the weights, and after passing through "transfer function", the output is obtained.

The output of the neuron in Figure 2 is obtained from equation 1. 


$$
Y=F\left(b+\sum_{i=1}^{N} W_{i}+X(i)\right)
$$

Figure 3 shows a three-layer neural network in which inputs are considered as the first layer. It is shown in $^{7}$ that three-layer neural networks are sufficient for modeling and approximation applications of continuous functions. The number of hidden layer neurons and the type of transfer functions are determined by the type, size, and complexity of the problem. The process of determining the weights in order to achieve optimal outputs in an artificial neural network is called neural network training. One of the most common methods for training multi-layer perception neural networks is the Back propagation method. As shown in Figure 4, this method uses the difference between neural network outputs and actual system outputs to adjust the weights to reduce this difference.

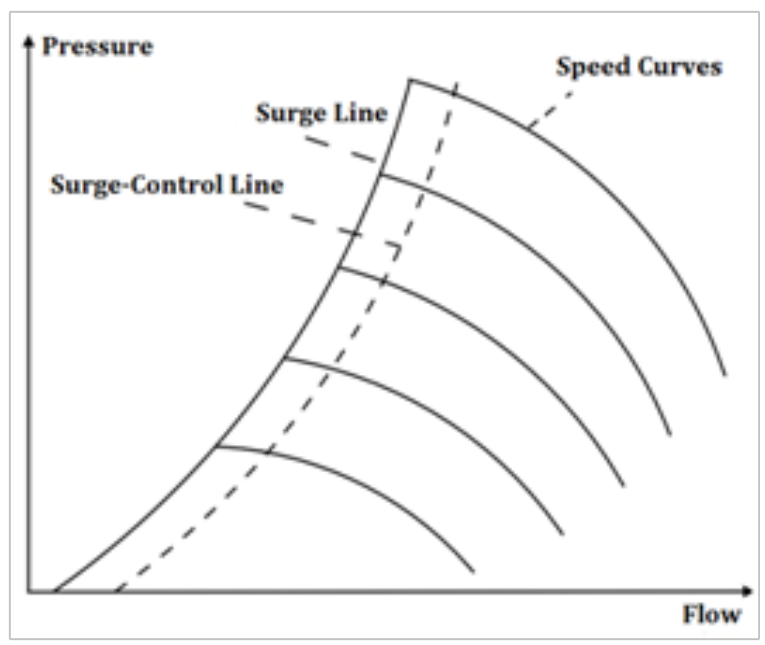

Figure I Indexed curves for a typical centrifugal compressor.

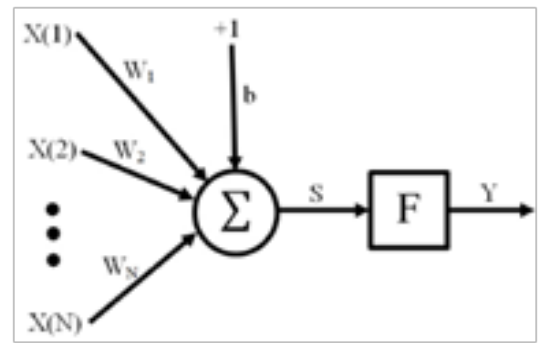

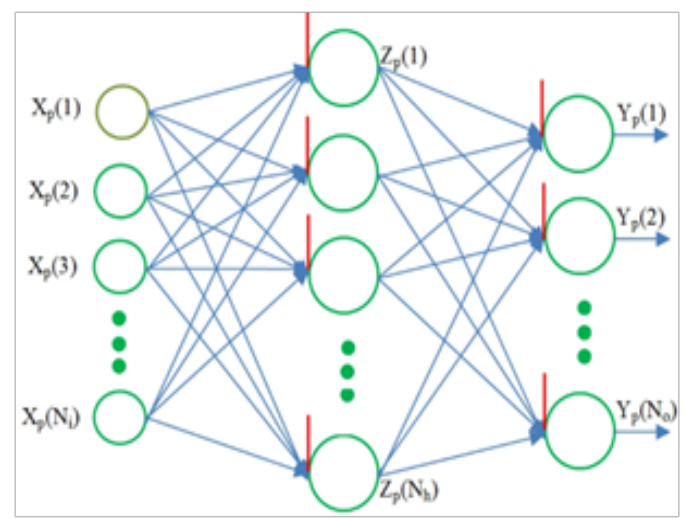

Figure 3 Perceptron neural network with three Layers.

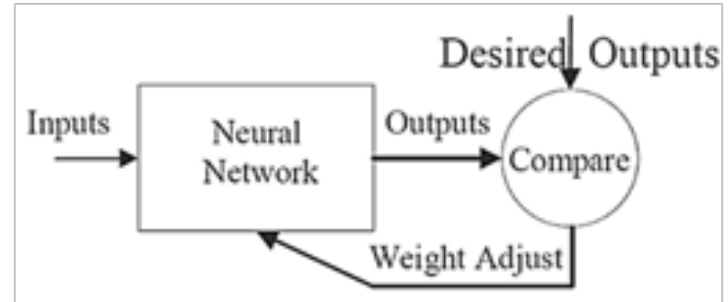

Figure 4 The procedure of neural network learning

A common cost function for assessment of neural network error is root mean squared error (RMSE) which is described as follow (equation 2):

$$
\text { RMSE }=\sqrt{\frac{1}{N_{s}} \sum_{i=1}^{N_{s}} e^{2}(i)}
$$

In the above statement, $\mathrm{N}_{\mathrm{s}}$ is the number of samples and e(i) is the prediction error for sample $i^{\text {th }}$.The sampled data from the actual system performance are divided into three parts: training set, validation set and test set. Training set is used for training the neural network, validation set is used for validating network performance during training, and test set is used to evaluate the neural network model obtained after training. All data is normalized in a specific range before applying to the neural network. Neural network outputs are non-normalized before being interpreted. This process is shown in Figure 5.

Figure 2 Artificial neuron.

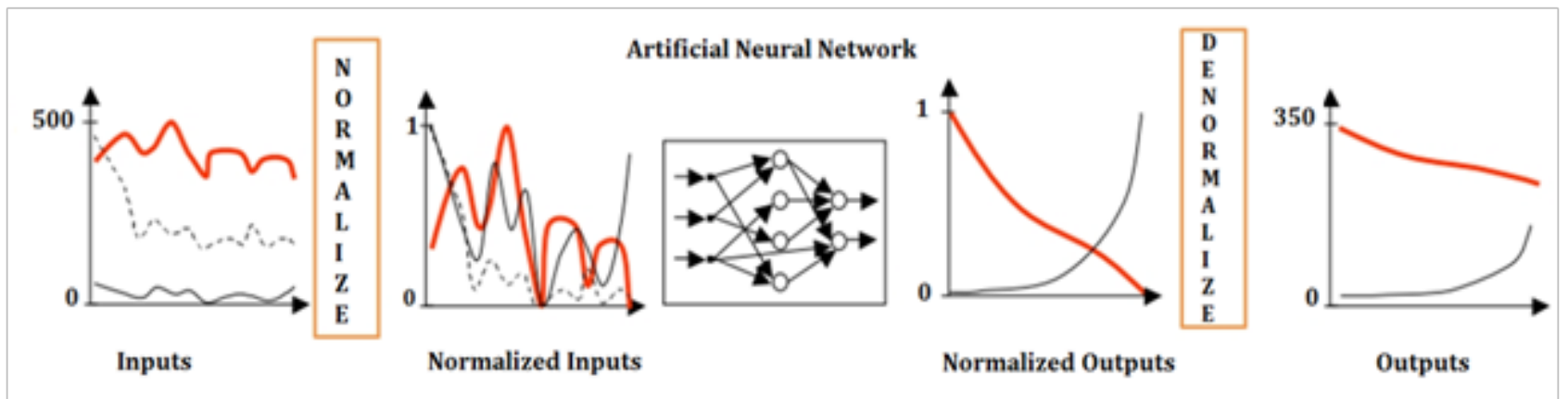

Figure 5 Pre \& post-processing procedures of data samples during ANN training. 


\section{Gas pressure boost system}

Figure 6 shows the studied gas pressure boost system at a natural gas processing plant. In this figure, the upstream tank is considered equivalent to the upstream compressor process. According to the measurements, the equivalent volume of the tank is $200 \mathrm{~m}^{3}$, and incoming gas having a constant flow of $8.5 \mathrm{~kg} / \mathrm{s} .{ }^{5}$ Gas from this tank enters the gas cooler of the shell and tube. In this cooling circuit, the cold fluid flow is regulated by a controller in such a way that the temperature of the output gas remains constant at about $25^{\circ} \mathrm{C}$. After this step, the gas enters the scrubber to separate the liquid from it. Gas pressure is then increased by a centrifugal compressor to about 85 bars. The return path, which is equipped with an anti-surge valve, allows the compressor to return the compressor gas to the cooling input to provide the required flow for the compressor. The part of the output gas that is not returned can be entered into the downstream tank of the compressor through a one-way value. This tank is considered equivalent to the downstream compressor process. Given that the compressor assumed in this project is only provide $3-5 \%$ of the flow of its downstream process, the gas pressure inside this tank is assumed to be approximately constant at 85 bars. A one-way valve prevents the downstream tank gas from returning to the compressor output. The torque required by the compressor is supplied by a gas turbine. The purpose of this paper is to provide an intelligent model based on artificial neural networks for investigating the effects of compressor RPM and the degree of openness of anti-surge valve on the temperature and flow rate of the compressor output gas and the gas pressure at compressor input as have shown in Figure 6.

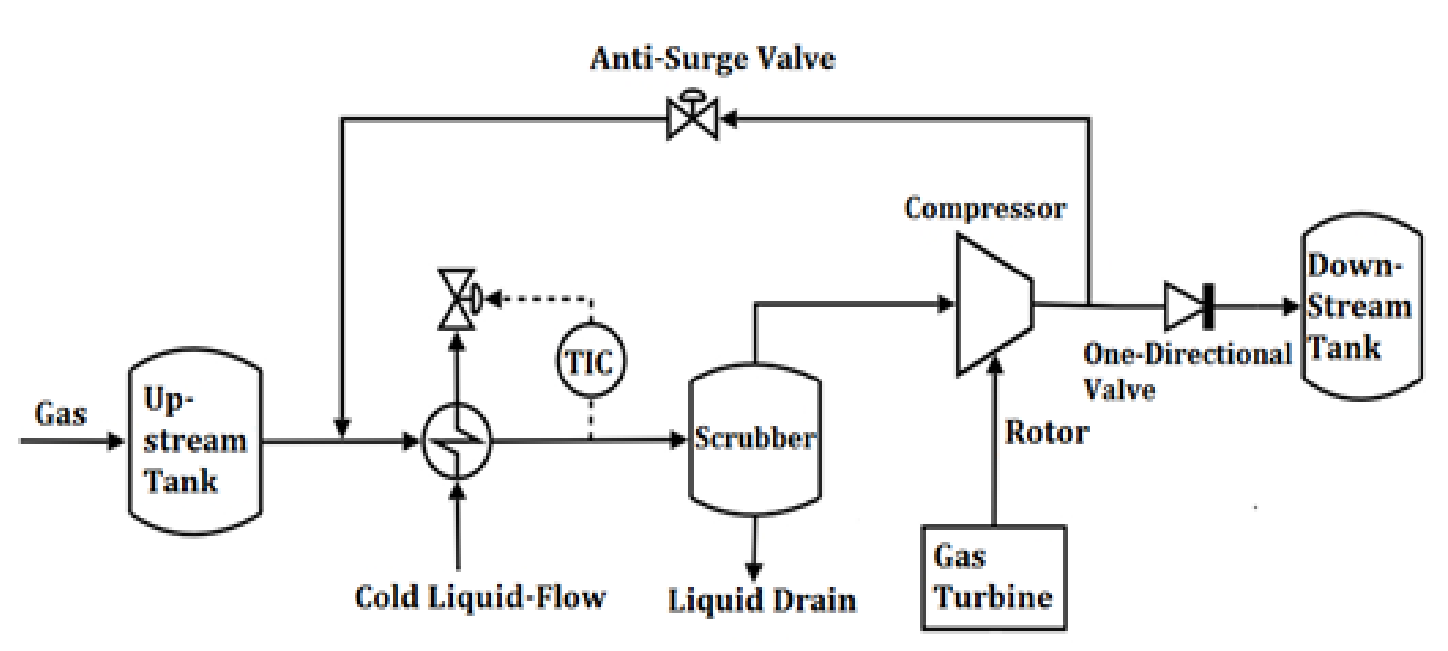

Figure 6 Gas Pressure Boost System.

\section{Results \& discussion}

The model presented in this paper is an artificial neural network of three-layer perception (Figure 3) with two inputs and three outputs. The network inputs are the compressor RPM and the degree of openness of the anti-surge valve and its outputs are the temperature, flow rate of compressor output gas and the gas pressure at the compressor input, respectively. All these parameters were measured in gas pressure boost system of Figure 6 by the cybernetics laboratory of Norway University in 16000 seconds at a sampling rate of 354 sample/minute. ${ }^{5}$ Thus, 93900 samples were obtained, of which $60 \%$ were considered as training set, $15 \%$ as validation set and $25 \%$ as test set. Data segmentation occurs at random every time the training process is repeated. To select the optimal number of hidden layer neurons and the type of transfer functions in each layer, we have used the simulation for different values of these parameters in Mat lab software. The simulations are performed for values ${ }^{2}$ as the number of hidden neurons (Nh). We also consider the three most commonly used modes for transfer functions: the first mode is using linear functions (purelin), the second mode is using the hyperbolic tangent functions (tansig), and the third mode is using the hyperbolic tangent functions for hidden layer neurons and linear functions for the output layer neurons (hybrid mode). To compare network performance in different situations, we have used the root mean square error (RMSE) and regression coefficient $(\mathrm{R})$ of output data. The results are shown in Table 1 .
Table I The effect of hidden-layer neuron numbers \& type of transfer function in ANN performance

\begin{tabular}{|c|c|c|c|c|c|c|c|}
\hline & & $\mathbf{N h}=\mathbf{I}$ & $\mathbf{N h}=2$ & Nh=3 & $\mathrm{Nh}=5$ & $N h=10$ & $\mathrm{Nh}=\mathbf{2 0}$ \\
\hline \multirow{3}{*}{ RMSE } & Purelin & 0.0098 & 0.007 & 0.007 & 0.007 & 0.007 & 0.007 \\
\hline & Tansig & 0.0094 & 0.0066 & 0.0062 & 0.0061 & 0.0055 & 0.0051 \\
\hline & Hybrid & 0.0096 & 0.0065 & 0.0062 & 0.0061 & 0.0054 & 0.005 \\
\hline \multirow{3}{*}{$R$} & Purelin & 0.9868 & 0.9933 & 0.9933 & 0.9933 & 0.9933 & 0.9933 \\
\hline & Tansig & 0.9879 & $0.994 I$ & 0.9947 & 0.9948 & 0.9958 & 0.9964 \\
\hline & Hybrid & 0.9873 & 0.9943 & 0.9947 & 0.9949 & 0.996 & 0.9965 \\
\hline
\end{tabular}

As can be seen, the best result is for a network with 20 neurons in its hidden layer with hyperbolic tangent transfer function for its hidden layer and linear transfer function for its output layer. However, it can be seen that except from the $\mathrm{Nh}=1$, in the remaining situations there is no significant difference between the efficiency of the network, so that even a very simple neural network with two hidden neurons and linear transfer functions can be very precise in predicting the desired outputs of the system. Simple networks with a low number of neurons occupy less memory space and require less processing time to generate outputs. Therefore, we consider the model of the neural network with a 2-2-3 structure and compare its outputs with real outputs and outputs derived from the model based on the state space equations proposed in. ${ }^{8}$ Data on the percentage of anti-surge 
valve openness and compressor speed are shown in Figures 7a, 7b, respectively. The actual and predicted values of the temperature and flow rate of the compressor output gas and its upstream pressure are shown in Figures 8a, 9a, 10a, respectively. Figures 8b, 9b, 10b also show the diagram and regression coefficient of each output. As can be seen, the neural network model presented in this paper is, in all cases, much better than the model based on state space equations.

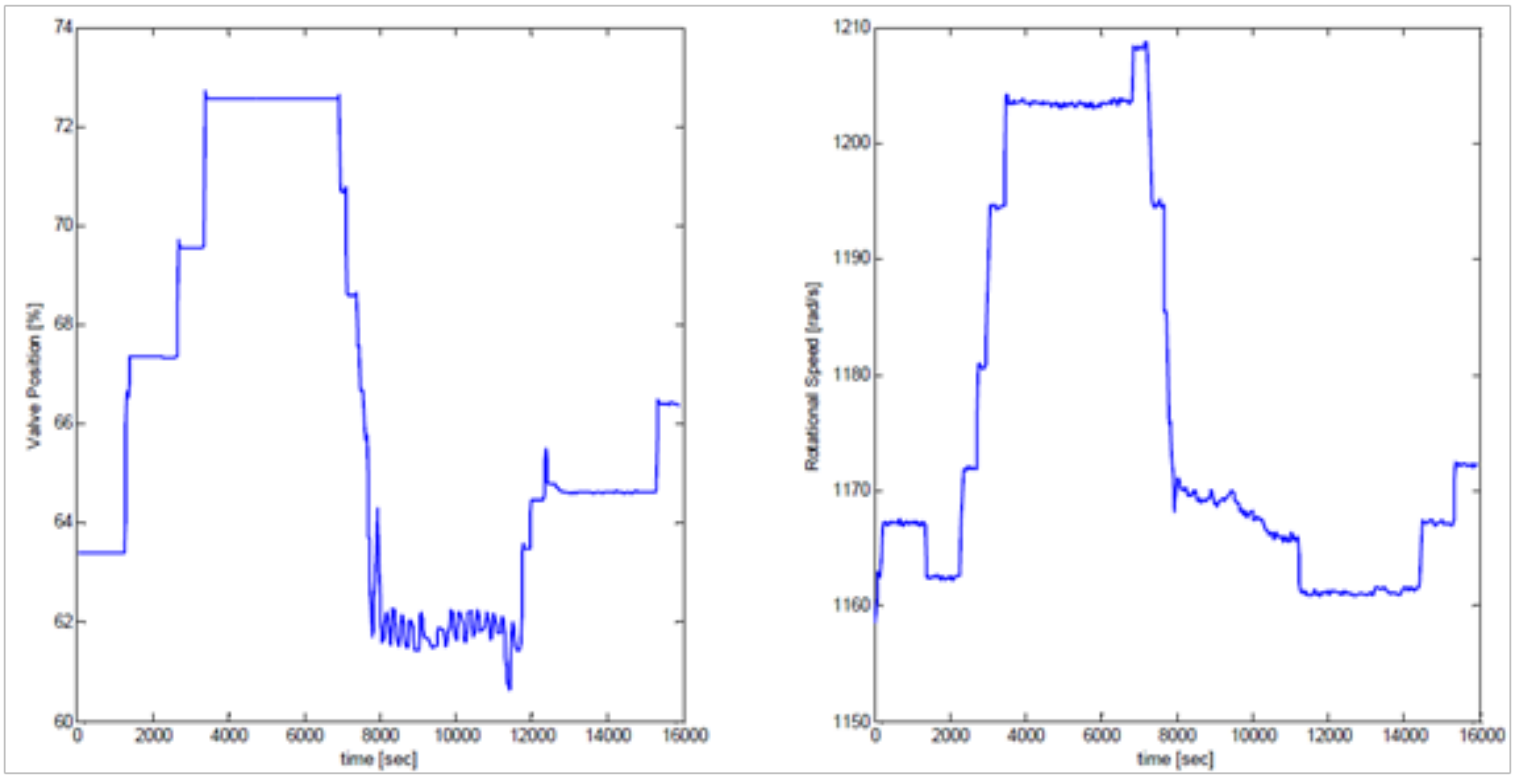

Figure 7 a) The percentage of anti-surge valve openness, and b) Compressor speed.
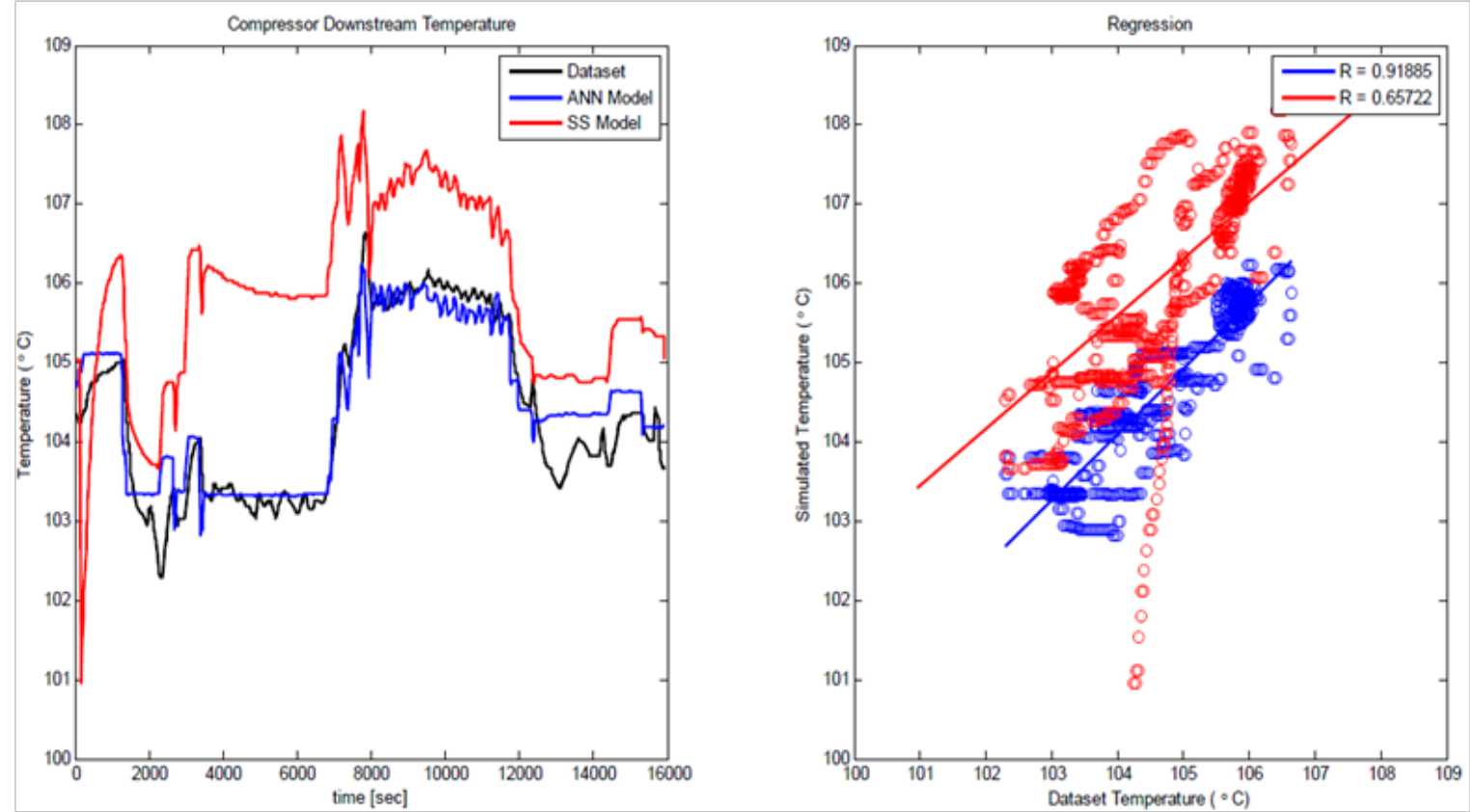

Figure 8 a) The temperature of the compressor output gas, and b) Temperature regression curve.

\section{Conclusion}

In this paper, we propose an artificial neural network model with 2 inputs and 3 outputs to investigate the effect of some of the effective parameters on the surge control in a centrifugal compressor used in a natural gas processing plant. The inputs of this model are, respectively, the compressor RPM and the anti-surge valve state, and its outputs are the temperature and flow rate of the compressor output and its upstream pressure. The data used in this paper were obtained from measurements conducted by cybernetics laboratory of Norway University in 16200 seconds at a sampling rate of 352sample/minutes. Of the 93900 available data, $60 \%$ were allocated to training set, $15 \%$ to validation set and $25 \%$ to test set. Then, by simulation, we have shown that a simple neural network with a 2-2-3 structure with linear transfer 
functions can predict the system outputs with acceptable accuracy. In addition, by presenting appropriate diagrams, we have shown that the accuracy and efficiency of the model presented in this paper is far better than the model based on state equations of the system, while the complexity of this model is much less than the model based on state equations of the system. In the future works, the effects of other parameters such as pressure changes in the compressor output, temperature changes at the input of the compressor, etc. should also be considered.
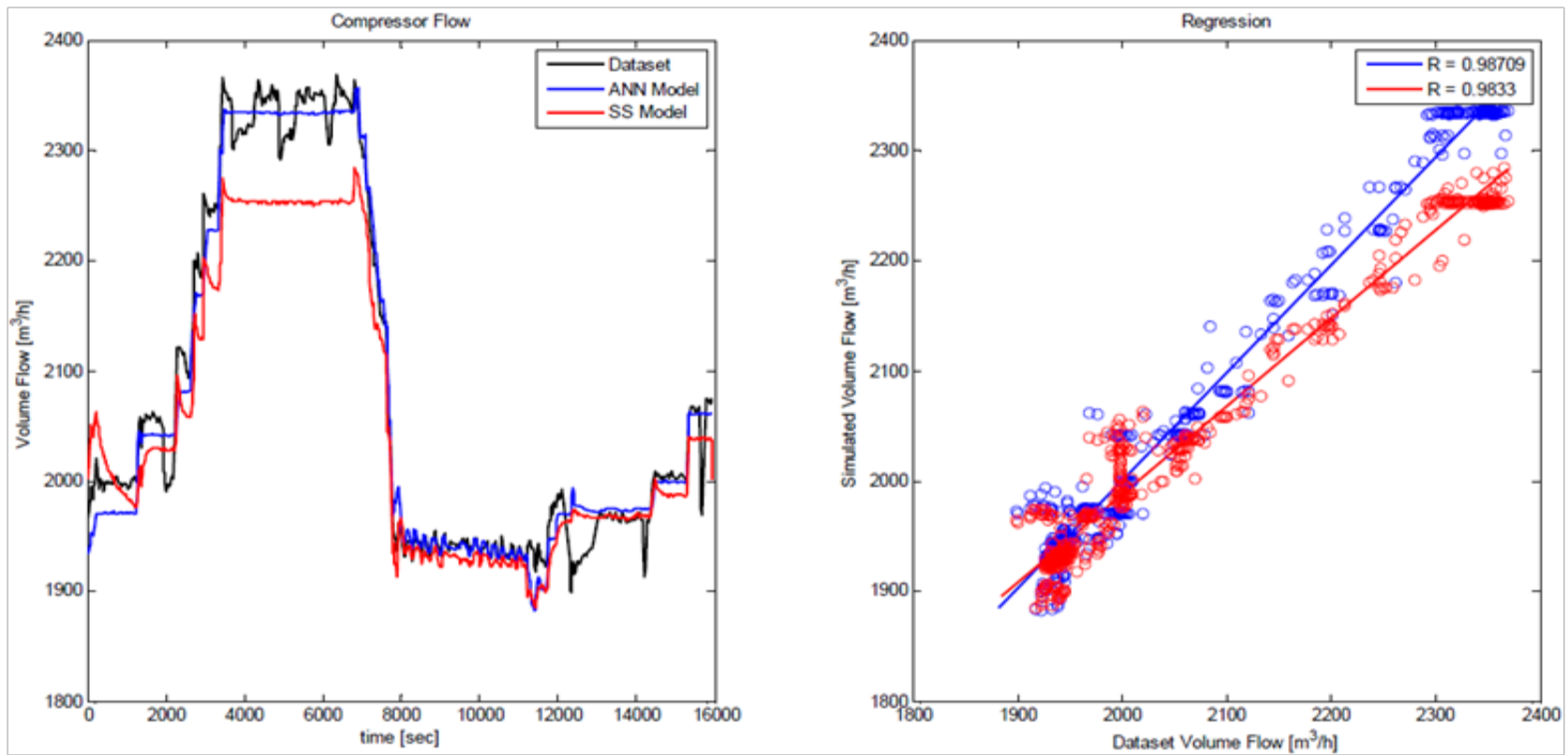

Figure 9 a) The Flow of the compressor output gas, and b) Flow regression curve.
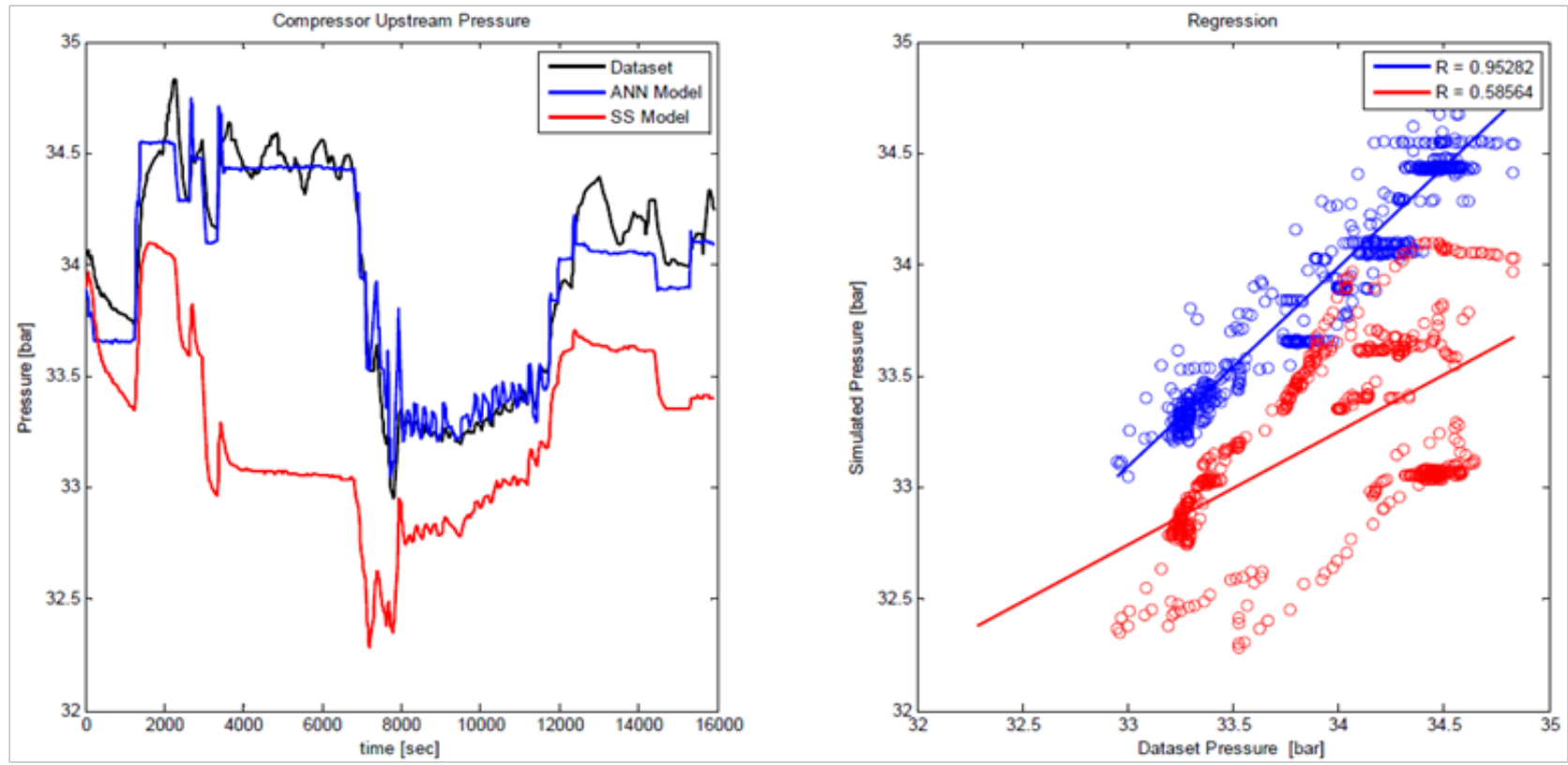

Figure 10 a) The Pressure of the compressor input gas, and b) Pressure regression curve.

\section{Acknowledgments}

No acknowledgement.

\section{Conflict of interest}

No conflict of interest.

\section{References}

1. Gravdahl JT, Egeland O. Compressor surge and rotating stall: modeling and control. Springer Science \& Business Media. 2012.

2. Torrisi G, Jaramillo V, Ottewill JR, et al. Active Surge control of electrically driven centrifugal compressors. Paper presented at the 2015 European Control Conference (ECC). 2015. 
3. Jiang W, Khan J, Dougal RA. Dynamic centrifugal compressor model for system simulation. Journal of Power Sources. 2006;158(2):1333-1343.

4. Cortinovis A, Pareschi D, Mercangoez M, et al. Model Predictive AntiSurge Control of Centrifugal Compressors with Variable-Speed Drives. IFAC Proceedings. 2012;45(8):251-256.

5. Zadeh Shirazi A, Mohammadi Z. A hybrid intelligent model combining ANN and imperialist competitive algorithm for prediction of corrosion rate in $3 \mathrm{C}$ steel under seawater environment. Neural Computing and Applications. 2016;28(11):3455-3464.
6. Zadeh Shirazi A, Hatami M, Yaghoobi M, et al. An Intelligent Approach to Predict Vibration Rate in a Real Gas Turbine. Intelligent Industrial Systems. 2016;2(3):253-267.

7. Zadeh Shirazi A, Seyyed Mahdavi Chabok SJ, Mohammadi Z. A novel and reliable computational intelligence system for breast cancer detection. Med Biol Eng Comput. 2017.

8. Aaslid P. Modelling of variable speed centrifugal compressors for antisurge control. 2009. 